\title{
COHOMOLOGY OF NILMANIFOLDS AND TORSION-FREE, NILPOTENT GROUPS
}

BY

\author{
LARRY A. LAMBE AND STEWART B. PRIDDY
}

\begin{abstract}
Let $M$ be a nilmanifold, i.e. $M=G / D$ where $G$ is a simply connected, nilpotent Lie group and $D$ is a discrete uniform, nilpotent subgroup. Then $M \simeq$ $K(D, 1)$. Now $D$ has the structure of an algebraic group and so has an associated algebraic group Lie algebra $L(D)$. The integral cohomology of $M$ is shown to be isomorphic to the Lie algebra cohomology of $L(D)$ except for some small primes depending on $D$. This gives an effective procedure for computing the cohomology of $M$ and therefore the group cohomology of $D$. The proof uses a version of form cohomology defined for subrings of $\mathbf{Q}$ and a type of Hirsch Lemma. Examples, including the important unipotent case, are also discussed.
\end{abstract}

Let $D$ be a finitely generated, torsion-free, nilpotent group of rank $n$. Then the upper central series of $D$ can be refined so that the $n$ successive subquotients are infinite cyclic. Thus $D \approx Z^{n}$ as sets and $P$. Hall $[\mathbf{H}]$ has shown that in these coordinates the product on $D$ is a polynomial function $\rho$. It follows that $D$ can be viewed as an algebraic group and so has an associated Lie algebra constructed from the degree two terms of $\rho$. The purpose of this paper is to study the integral cohomology of $D$ using this algebraic group Lie algebra.

Although these notions are purely algebraic, it is helpful to work in a more geometric context using A. Malcev's equivalence [M] between the category of finitely generated, torsion-free, nilpotent groups and the category of nilmanifolds; i.e., compact manifolds of the form $M=G / D$ where $G$ is a simply connected nilpotent Lie group and $D$ is a discrete subgroup. It follows that $D$ is nilpotent, finitely generated and torsion-free. Moreover, $M$ is an Eilenberg-Mac Lane space $K(D, 1)$. Conversely given such a $D$, Malcev has constructed a simply connected nilpotent Lie group $D_{\mathbf{R}}$ such that $D_{\mathbf{R}} / D$ is a nilmanifold.

In 1954, K. Nomizu [N] established an isomorphism

$$
H^{*}(G / D ; \mathbf{R}) \approx H^{*}(\mathcal{L}(G))
$$

between the real cohomology of $G / D$ and the cohomology of the classical Lie algebra $\mathcal{L}(G)$ of $G$. In seeking to extend this result to include torsion, we replace $\mathcal{L}(G)$ by its generalization, the algebraic group Lie algebra (1.8) of $D$. Our main

Received by the editors April 29, 1981 and, in revised form, November 2, 1981. Presented by the first author at the January 1981 meeting of the American Mathematical Society at San Francisco in the special session on homotopy theory.

1980 Mathematics Subject Classification. Primary 20J05, 55T15, 58A12, 22E12, 22 E40.

Key words and phrases. Nilmanifolds, torsion-free, nilpotent groups, formal groups, de Rham cohomology.

(C)1982 American Mathematical Society 0002-9947/81/0000-0248/\$06.25 
result, Theorem 4.1, then says that for a large class of groups $D$, Nomizu's result continues to hold integrally except for certain initial primes for which we give a bound depending on $D$.

Nomizu's proof uses de Rham cohomology of differential forms and generalizes to rational cohomology using Sullivan's theory [Su]. The analogous integral version of de Rham cohomology developed by H. Cartan [C] and E. Miller [Mi] uses the forms with a certain grading depending on the "size" of the form; unfortunately the forms of a nilmanifold are not graded in this sense. This is the basic difficulty which forces us to use a filtered version of form cohomology defined over subrings $\mathbf{Q}_{r}=$ $\mathbf{Z}\left[\frac{1}{2}, \frac{1}{3}, \ldots, \frac{1}{r}\right]$ of $\mathbf{Q}$. Cenkl and Porter [CP] have developed such a theory for cubical cohomology but for our purposes it is more useful to modify Cartan's theory since it holds for an arbitrary simplicial set. Another difficulty is the lack, in general, of a natural integral Lie algebra associated with $D$. There are, of course, numerous graded Lie algebras associated with various filtrations of $D$, but these can only be expected to relate (via spectral sequences) to an associated graded version of the cohomology of $D$.

The paper is organized as follows: The basic structure of nilmanifolds and torsion-free, nilpotent groups is described in $\$ 1$. The algebraic group Lie algebra is contructed as well as other Lie algebras used in the paper. An explicit formula for the transgression is given when a nilmanifold is expressed as a sequence of principle $S^{1}$-bundles. In $\$ 2$, we exhibit the invariant differential forms and vector fields associated with a nilmanifold. The chain complex of right invariant forms is seen to coincide with the chain complex for computing the cohomology of the algebraic group Lie algebra. A version of form cohomology with coefficients in $\mathbf{Z}\left[\frac{1}{2}, \frac{1}{3}, \ldots, \frac{1}{r}\right]$ is developed in $\S 3$. In $\S 4$, we prove the main Theorem 4.1 by induction on the rank of $D$, using the theory of $\$ 3$ and a type of Hirsch Lemma. In $\$ 5$ we show how choices of canonical bases give rise to naturality in the isomorphism of 4.1 . $\$ 6$ is devoted to calculations and examples including the important unipotent groups. Finally in $§ 7$, we consider other Lie algebras which one may associate to torsion-free, nilpotent groups.

1. Structure of nilmanifolds. In this section we record some fundamental facts about nilmanifolds, torsion-free, nilpotent groups, and their associated Lie algebras. Basic references are Malcev [M], P. Hall [H] and Serre [S].

1.0 Definition. A nilmanifold is a compact manifold of the form $M=G / D$ where $G$ is a simply connected nilpotent Lie group and $D$ is a discrete subgroup. It follows that $D$ is finitely generated, torsion-free and nilpotent [M].

1.1 ExAmple. A good generic example is $G=U_{n}(\mathbf{R})$, the unipotent group over $\mathbf{R}$ (upper triangular matrices with ones down the diagonal). In this case $D=U_{n}(\mathbf{Z})$.

1.2 Exponential map. The exponential map for $G$ is a global diffeomorphism, i.e. $G$ is a euclidian space. Thus $M$ is a $K(D, 1)$ with $D=\pi_{1} M$.

1.3 Rank. There is a refinement $D=D_{1} \supset D_{2} \supset \cdots \supset D_{n} \supset D_{n+1}=1$ of the upper central series of $D$ with $D_{i} / D_{i+1} \approx \mathbf{Z}$ for all $i$. The integer $n$ is called the rank and is an invariant of $D[\mathbf{H}]$.

1.4 Canonical basis. If $\left\{u_{1}, \ldots, u_{n}\right\} \subset D$ is chosen so that $D_{i}=\left\langle D_{i+1}, u_{i}\right\rangle$ then each element of $D$ is uniquely expressible as $u_{1}^{x_{1}} u_{2}^{x_{n}} \cdots u_{n}^{x_{n}}, x_{i} \in \mathbf{Z}$. The set 
$\left\{u_{1}, \ldots, u_{n}\right\}$ is called a canonical or Malcev basis and a choice of such basis defines an isomorphism $D \approx \mathbf{Z}^{n}$ as sets [H].

1.5 Polynomial multiplication. Using a canonical basis the multiplication on $D$ takes the form

$$
u_{1}^{x_{1}} u_{2}^{x_{2}} \cdots u_{n}^{x_{n}} \cdot u_{1}^{y_{1}} u_{2}^{y_{2}} \cdots u_{n}^{y_{n}}=u_{1}^{\rho_{1}(x, y)} u_{2}^{\rho_{2}(x, y)} \cdots u_{n}^{\rho_{n}(x, y)}
$$

where $\rho_{i}: \mathbf{Z}^{n} \times \mathbf{Z}^{n} \rightarrow \mathbf{Z}$ is a polynomial function over $\mathbf{Q}$ for $1 \leqslant i \leqslant n$. Furthermore, [H]

$$
\rho_{i}(x, y)=x_{i}+y_{i}+\tau_{i}\left(x_{1}, \ldots, x_{i-1}, y_{1}, \ldots, y_{i-1}\right) .
$$

EXAMPLE. In the unipotent case, $D=U_{n}(\mathbf{Z})$ has canonical basis $\left\{u_{i j}: 1 \leqslant i<j \leqslant n\right\}$ where $u_{i j}$ agrees with the identity matrix, except the $(i, j)$ th entry is 1 . In this basis $D \approx\left(\mathbf{Z}^{N}, \rho\right)$ where $N=n(n-1) / 2$ and $\rho_{i j}(x, y)=x_{i j}+y_{i j}+\sum_{i<k<j} x_{i k} y_{k j}$.

REMARK. It is known [St] that every polynomial function $f: \mathbf{Z}^{n} \rightarrow \mathbf{Z}$ in $\mathbf{C}\left[x_{1}, \ldots, x_{n}\right]$ is an integral linear combination of monomials

$$
\left(\begin{array}{c}
x_{i_{1}} \\
r_{i_{1}}
\end{array}\right)\left(\begin{array}{c}
x_{i_{2}} \\
r_{i_{2}}
\end{array}\right) \cdots\left(\begin{array}{c}
x_{i_{j}} \\
r_{i_{j}}
\end{array}\right)
$$

where $1 \leqslant i_{k} \leqslant m$ and $r_{i_{k}} \geqslant 0$ are integers satisfying $r_{i_{k}} \leqslant$ degree of $x_{i_{j}}$ in $f$. For example, let $D$ be the group (given in terms of generators and relations)

$$
D=\left\langle u_{1}, u_{2}, u_{3}, u_{4}:\left(u_{1}, u_{2}\right)=u_{3},\left(u_{1}, u_{3}\right)=u_{4},\left(u_{2}, u_{3}\right)=1,\left(u_{i}, u_{4}\right)=1\right\rangle
$$

where $(x, y)=x^{-1} y^{-1} x y$. Then $D$ is a finitely generated, torsion-free, nilpotent group and $\left\{u_{1}, \ldots, u_{4}\right\}$ is a canonical basis. Using the relations one can easily see that the corresponding $\rho_{i}$ are

$$
\begin{aligned}
& \rho_{i}(x, y)=x_{i}+y_{i}, \quad i=1,2, \\
& \rho_{3}(x, y)=x_{3}+y_{3}-x_{2} y_{1}, \\
& \rho_{4}(x, y)=x_{4}+y_{4}-x_{3} y_{1}+x_{2}\left(\begin{array}{c}
y_{1} \\
2
\end{array}\right) .
\end{aligned}
$$

1.6 Extension of rings. A choice of canonical basis defines a group isomorphism $D \approx\left(\mathbf{Z}^{n}, \rho\right)$ where $\rho=\left(\rho_{1}, \rho_{2}, \ldots, \rho_{n}\right)$. Thus by extending the domain of $\rho$ we may define $D_{R}=\left(R^{n}, \rho\right)$ for various rings $R$. For example this makes sense for $R \subset \mathbf{Q}$ or $R=\mathbf{R}$ since in these cases $r \in R, m \in \mathbf{Z}$ implies $\left(\begin{array}{l}r \\ m\end{array}\right) \in R$. Since the group axioms for $\left(\mathbf{Z}^{n}, \rho\right)$ express themselves as polynomial relations, $D_{R}$ is also a group. For $R=\mathbf{Q}, D_{R}$ is the localization at 0 or rationalization and for $R=\mathbf{R}, D_{R}$ is the real completion. In 1.1, $U_{n}(\mathbf{Z})_{\mathbf{Q}}=U_{n}(\mathbf{Q})$ and $U_{n}(\mathbf{Z})_{\mathbf{R}}=U_{n}(\mathbf{R})$.

1.7 The nilmanifold associated to $D$. Given a finitely generated, torsion-free, nilpotent group $D$, then $D$ is a subgroup of the simply connected Lie group $D_{\mathbf{R}}$ and $M=D_{\mathbf{R}} / D$ is a nilmanifold. Conversely if we are given a nilmanifold $M=G / D$ then $G \approx D_{\mathbf{R}}$ as Lie groups and $M$ is diffeomorphic to $D_{\mathbf{R}} / D$.

1.8 Algebraic group Lie algebras. Let $R \subset \mathbf{Q}$ be the subring generated by the coefficients of $\rho$, where $D \approx\left(Z^{n}, \rho\right)$ as in 1.6. By considering $\rho_{i}$ as an element of $R\left[x_{1}, \ldots, x_{n}, y_{1}, \ldots, y_{n}\right]$ we see that a choice of canonical basis gives $R^{n}$ the structure of an affine algebraic group over $R$ [Hu, §7]. In general, if $R$ is any commutative ring 
with 1 , such a structure consists of an $n$-tuple $\rho=\left(\rho_{1}, \ldots, \rho_{n}\right)$ where $\rho_{i} \in$ $R\left[x_{1}, \ldots, x_{n}, y_{1}, \ldots, y_{n}\right]$ such that

1. $\rho(x, 0)=\rho(0, x)=x(0$ is the identity),

2. $\rho(\rho(x, y), z)=\rho(x, \rho(y, z))$ (associative law).

In this case there is a unique $\Phi \in R\left[x_{1}, \ldots, x_{n}\right]$ such that $\Phi(0)=0$ and

3. $\rho(\Phi(x), x)=\rho(x, \Phi(x))=0$ (unique inverse).

If we set $[x, y]=b(x, y)-b(y, x)$ where $b$ is the homogeneous degree 2 term of $\rho$ then $\rho(x, y)=x+y+b(x, y)+$ terms of degree $\geqslant 3$ and $[\cdot, \cdot]$ satisfies the Jacobi identity

$$
[x,[y, z]]+[y,[x, z]]+[z,[x, y]]=0 .
$$

Thus any such $\rho$ over $R$ gives rise to an associated algebraic group Lie algebra, which, in our case, we denote by $L_{R}(D)$. The underlying $R$-module structure of $L_{R}(D)$ is $R^{n}=R \oplus \cdots \oplus R$ with bracket as defined above. Since we use none of the theory of algebraic groups, the reader could equally well consider these objects as formal groups and formal Lie algebras respectively. A concise treatment of their properties appears in Serre [S, LG 18].

When the coefficients of $\rho$ are integers (e.g. if $D=U_{n}(\mathbf{Z})$ ) then we obtain an integral Lie algebra. In any case tensoring with $\mathbf{Q}$ and $\mathbf{R}$ we have the rational Lie algebra $L_{\mathbf{Q}}(D)=\left(\mathbf{Q}^{n},[\cdot, \cdot]\right)$ and the real Lie algebra $L_{\mathbf{R}}(D)=\left(\mathbf{R}^{n},[\cdot, \cdot]\right)$. Since the nilmanifold $M=D_{\mathbf{R}} / D$ is determined by $D$ all of these Lie algebras could equally well be thought of as associated to $M$. This point is clarified in 1.9.

1.9 Relations with the Lie group Lie algebra. Let $\mathfrak{X}(N)$ denote the vector fields on a manifold $N$ and let $G=D_{\mathbf{R}}$. Then the classical Lie algebra $\mathscr{L}(G)$ can be identified with $\mathfrak{X}_{G}(G)$, the right-invariant vector fields on $G$.

Lemma. $\mathcal{L}(G) \approx L_{\mathbf{R}}(D)$ as Lie algebras.

Proof. Let $T_{1}(G)$ denote the tangent space at the identity. If $X$ is a vector field, let $X_{1}$ denote its value at 1 . Then $X \mapsto X_{1}$ defines an isomorphism $\mathcal{L}(G) \stackrel{\approx}{\rightarrow} T_{1}(G)$ with inverse $v \mapsto \tilde{v}$ where $\tilde{v}(f)(g)=v\left(f \circ r_{g}\right)$ and $r_{g}: G \rightarrow G$ is right multiplication by $g$. Now $G$ is diffeomorphic to $\mathbf{R}^{n}$ (see 1.2) so $\mathfrak{X}_{G}(G) \subset \mathfrak{X}(G) \approx \mathfrak{X}\left(\mathbf{R}^{n}\right.$ ) and $T_{1}(G) \approx T_{0}\left(\mathbf{R}^{n}\right)$. Thus $\mathfrak{X}_{G}(G)$ has a basis $\left\{E_{1}, \ldots, E_{n}\right\}$ where

$$
E_{j}(f)(x)=\left.\left(\partial / \partial y_{j}\right)\right|_{0}\left(f \circ r_{x}\right) .
$$

If we write $E_{j}=\sum_{i=1}^{n} a_{i j}\left(\partial / \partial x_{i}\right), a_{i j} \in C^{\infty}(G)$, and let $e^{i}: \mathbf{R}^{n} \rightarrow \mathbf{R}$ denote the $i$ th coordinate function then

$$
a_{i j}(x)=E_{j}\left(e^{i}\right)(x)=\left.\frac{\partial}{\partial y_{j}}\right|_{0}\left(e^{i} \circ r_{x}\right)=\left.\frac{\partial}{\partial y_{j}}\right|_{0} \rho_{i}(y, x) .
$$

Thus

$$
\left[E_{i}, E_{j}\right]=\sum_{k, s}\left(a_{s i} \frac{\partial a_{k j}}{\partial x_{i}}-a_{s j} \frac{\partial a_{k i}}{\partial x_{j}}\right) \frac{\partial}{\partial x_{k}}
$$

and evaluating at 0 we get

$$
\left[E_{i}, E_{j}\right]_{0}=\left.\sum_{k}\left(\frac{\partial a_{k j}}{\partial x_{i}}(0)-\frac{\partial a_{k i}}{\partial x_{j}}(0)\right) \frac{\partial}{\partial x_{k}}\right|_{0} .
$$


However $\left(\partial a_{k j} / \partial x_{i}\right)(0)=\left.\left.\left(\partial / \partial x_{i}\right)\right|_{0}\left(\partial / \partial y_{j}\right)\right|_{0} \rho_{k}(y, x)=b_{k}\left(e_{j}, e_{i}\right)$ where $e_{k}$ denotes the standard basis vector and $b(x, y)=\left(b_{1}(x, y), \ldots, b_{n}(x, y)\right)$ is the homogeneous degree two part of $\rho$. Thus $\mathcal{L}(G)$ and $L_{\mathbf{R}}(D)$ have the same structure constants up to a minus sign.

1.10 Extension cocycle. Consider $D \approx\left(\mathbf{Z}^{n}, \rho\right)$ as in 1.6. Let $z=\left\langle e_{n}\right\rangle$ where $e_{n}=(0, \ldots, 1)$. Then $Z \approx Z$ and we have a central extension

$$
1 \rightarrow Z \rightarrow D \rightarrow \bar{D} \rightarrow 1
$$

where $\bar{D}$ is obtained by projecting the last coordinate of $\mathbf{Z}^{n}$ to 0 , i.e. for $x, y \in \mathbf{Z}^{n-1}$

$$
\overline{\rho_{i}}(x, y)=\rho_{i}((x, 0),(y, 0))=x_{i}+y_{i}+\tau_{i}\left(x_{1}, \ldots, x_{i-1}, y_{1}, \ldots, y_{i-1}\right),
$$

$1 \leqslant i \leqslant n-1$ (see 1.5). Furthermore $\bar{D} \times \bar{D}^{\tau_{n}} \rightarrow \mathbf{Z}$ is the cocycle of this extension.

1.11 Nilmanifolds and principal $S^{1}$-bundles. From 1.10 we obtain the principal $S^{1}$-bundle

$$
\begin{aligned}
s^{1}=\mathbf{R} / \mathbf{Z} \rightarrow & D_{\mathbf{R}} / D \\
& \downarrow \\
& \bar{D}_{\mathbf{R}} / \bar{D}
\end{aligned}
$$

classified by $\tau_{n}: \bar{D}_{\mathbf{R}} / \bar{D} \rightarrow K(\mathbf{Z}, 2)$, where we identify $H^{2}\left(\bar{D}_{\mathbf{R}} / \bar{D} ; \mathbf{Z}\right)$ with the group cohomology $H^{2}(\bar{D} ; \mathbf{Z})$. Iterating this procedure decomposes the nilmanifold $M=$ $D_{\mathbf{R}} / D$ as a tower of principal fibrations

$$
\begin{array}{ccccc}
S^{1} & \rightarrow & M_{n}= & M & \\
& & \downarrow & & \\
S^{1} & \rightarrow & M_{n-1} & \stackrel{\tau_{n}}{\rightarrow} & K(\mathbf{Z}, 2) \\
& & \downarrow & & \\
& & \vdots & & \\
& & \downarrow & & \\
S^{1} & \rightarrow & M_{2} & \stackrel{\tau_{3}}{\rightarrow} & K(\mathbf{Z}, 2) \\
& & \rightarrow & & \\
S^{1} & \rightarrow & M_{1} & \stackrel{\tau_{2}}{\rightarrow} & K(\mathbf{Z}, 2) \\
& & \downarrow & & \\
& & * & \stackrel{\tau_{1}}{\rightarrow} & K(\mathbf{Z}, 2) .
\end{array}
$$

Conversely given such a tower we obtain a finitely generated, torsion-free, nilpotent group $D=\pi_{1}(M)$. It is also clear that $D_{i}=\pi_{1}\left(M_{n+1-i}\right)$ is the refinement of the upper central series of $D$ of 1.3 .

Remark. In theory, $H^{*}(M)$ can be computed from tower (1) since the Serre spectral sequence for a circle fibration reduces to a simple Gysin sequence. In practice, however, one encounters multiplicative extension problems very quickly and the process breaks down. Hirsch [Hi] has shown this phenomenon is related to nontrivial Massey products. In a sense, the main result of this paper (Theorem 4.1) 
says that under certain conditions the $E_{2}$ terms of these Serre spectral sequences can be combined into a single complex for computing $H^{*}(M)$.

2. Invariant forms and Lie algebra cohomology. For the remainder of this paper we shall assume that $D$ is a finitely generated, torsion-free, nilpotent group of rank $n$ and that by a choice of canonical basis we have $D \approx\left(\mathrm{Z}^{n}, \rho\right)$ as in 1.4 and 1.5. Then $G=D_{\mathbf{R}}$ is the Lie group obtained by completion as in 1.6 so that $M=G / D$ is a nilmanifold.

In this section we determine the right invariant forms $\Lambda_{G}^{*}(G)$ on $G$. It is classical that the chain complex $\Lambda_{G}^{*}(G)$ computes the real cohomology of the Lie algebra $\mathcal{L}(G)$. We show that after extending the ground ring, the chain complex for computing the cohomology of the algebraic group Lie algebra $L_{R}(D)$ of 1.8 coincides with $\Lambda_{G}^{*}(G)$.

2.1 Invariant forms. Let $E_{j}=\sum_{i=1}^{n} a_{i j}\left(\partial / \partial x_{i}\right)$, where $a_{i j}(x)=\left.\left(\partial / \partial y_{j}\right)\right|_{0}\left(\rho_{i}(y, x)\right)$. We have seen (1.9) that $\left\{E_{i}\right\}_{i=1}^{n}$ is a basis for the right invariant vector fields $\mathfrak{X}_{G}(G)$ on $G$. The matrix $A=\left(a_{i j}\right)$ of $C^{\infty}(G)$ functions is upper unitriangular, in fact, from the expression for $\rho$ in 1.5 we have

$$
\begin{aligned}
a_{i j}(x) & =\left.\frac{\partial}{\partial y_{j}}\right|_{0}\left(\rho_{i}(y, x)\right) \\
& = \begin{cases}0 \quad \text { if } i>j, \\
1 \quad \text { if } i=j, \\
\left.\frac{\partial}{\partial y_{j}}\right|_{0}\left(y_{i}+x_{i}+\tau_{i}\left(y_{1}, \ldots, y_{i-1}, x_{1}, \ldots, x_{i-1}\right)\right) \text { if } i<j .\end{cases}
\end{aligned}
$$

Thus $E_{j}=\partial / \partial x_{j}+a_{j, j+1}\left(\partial / \partial x_{j+1}\right)+\cdots+a_{j, n}\left(\partial / \partial x_{n}\right)$. Now consider the 1-forms on $G$ defined by

$$
\varepsilon_{j}=\sum_{i=1}^{n} \omega_{i j} d x_{i}
$$

where $\omega_{i j}(x)=\left.\left(\partial / \partial y_{i}\right)\right|_{0}\left(\rho_{j}\left(y, x^{-1}\right)\right)$. This is the right invariant 1 -form whose value at $x$ is given by $\varepsilon_{j}(x)=r_{x^{-1}}^{*}\left(d x^{j}\right)$ where $r_{x^{-1}}$ is right multiplication by $x^{-1}$. Then $\left\{\varepsilon_{j}\right\}_{j=1}^{n}$ is a basis for $\Lambda_{G}^{1}(G)$, the right invariant 1-forms of $G$. Furthermore if $W=\left(\omega_{i j}\right)$ then $W=\left(A^{-1}\right)^{\mathrm{t}}$ and so $W$ is lower unitriangular

$$
\varepsilon_{j}=\omega_{1 j} d x_{1}+\omega_{2 j} d x_{2}+\cdots+\omega_{j-1, j} d x_{j-1}+d x_{j} .
$$

2.2 Descent. $\left(\mathbf{Z}^{n}, \rho\right)$ has canonical basis $\left\{e_{i}\right\}_{i=1}^{n}$. Let $R$ be the subring of $\mathbf{Q}$ generated by the coefficients of $\rho$ as in 1.8. Then $L_{R}(D)=\left(R^{n},[\cdot, \cdot]\right)$ has structure constants

$$
\left[e_{i}, e_{j}\right]=\sum_{k=1}^{n} c_{i, j}^{k} e_{k}
$$

where $c_{i, j}^{k}=b_{k}\left(e_{i}, e_{j}\right)-b_{k}\left(e_{j}, e_{i}\right)$. Dualizing we have the Koszul complex $\left(\Lambda^{*}\left(L_{R}(D)^{*}\right), d\right)$, a DGA with $\Lambda^{k}\left(L_{R}(D)^{*}\right)$ the $k$ th exterior power of the dual space $L_{R}(D)^{*}=\operatorname{Hom}_{R}\left(L_{R}(D), R\right)$. The differential is given by

$$
d e^{k}=\sum_{1 \leqslant i<j \leqslant n} c_{i, j}^{k} e^{i} \wedge e^{j}
$$


where $\left\{e^{i}\right\}_{i=1}^{n}$ is the dual basis to $\left\{e_{j}\right\}$ and $d$ is extended as a derivation. The homology of $\left(\Lambda^{*}\left(L_{R}(D)^{*}\right), d\right)$ is the cohomology of the Lie algebra $L_{R}(D)$ [CE].

The structure constants of the Lie algebra $\mathcal{L}(G)=\mathfrak{X}_{G}(G)$ in the basis $\left\{E_{j}\right\}$ are the $c_{i, j}^{k}$ (see 1.9). In this sense we have a reduction of the Lie algebra to $R$. Furthermore, if $\left(\Lambda^{*}(G), d\right)$ denotes the de Rham complex of $G$ then $\left(\Lambda_{G}^{*}(G), d\right)$ is a subcomplex consisting of the right invariant forms on $G$. Choosing the basis $\left\{\varepsilon_{i}\right\}$ of 2.1 we have that $d$ is the unique extension of

$$
d \varepsilon_{K}=\sum_{1 \leqslant i<j \leqslant n} c_{i, j}^{k} \varepsilon_{i} \wedge \varepsilon_{j}
$$

as a derivation where the $c_{i, j}^{k}$ are the same as those above. This is the classical Maurer-Cartan formula $[\mathrm{Cv}]$. In this sense we have the reduction of $\left(\Lambda_{G}^{*}(G), d\right)$ to the ring $R$ which we denote by $\left(\Lambda^{*}(G: R), d\right)$, and an isomorphism of DGA's over $R$,

$$
\left(\Lambda^{*}(G: R), d\right) \approx\left(\Lambda^{*}\left(L_{R}(D)^{*}\right), d\right) .
$$

2.3 Form weight. It will be useful in subsequent sections to assign to each $k$-form $\omega \in \Lambda_{G}^{k}(G)$, an integer which reflects its polynomial weight. By 2.1 the basis $\left\{\varepsilon_{j}\right\}$ for $\Lambda_{G}^{1}(G)$ consists of forms with polynomial coefficients

$$
\varepsilon_{j}=\omega_{1 j} d x_{1}+\omega_{2 j} d x_{2}+\cdots+\omega_{j-1, j} d x_{j-1}+d x_{j}
$$

$\omega_{i j} \in R\left[x_{1}, \ldots, x_{n}\right]$. Thus the basis $\varepsilon_{i_{1}} \wedge \cdots \wedge \varepsilon_{i_{l}}$ for $\Lambda_{G}^{l}(G)$ consists of forms with polynomial coefficients, say

$$
\omega=\varepsilon_{i_{1}} \wedge \cdots \wedge \varepsilon_{i_{l}}=\sum p_{j}\left(x_{1}, \ldots, x_{n}\right) d x_{j_{1}} \wedge \cdots \wedge d x_{j_{l}} .
$$

The form weight $s$ of $\omega$ is $s=k+l$ where $k$ is the largest polynomial degree of the $p_{j}\left(x_{1}, \ldots, x_{n}\right)$. The maximum form weight of $G$ with respect to $\left(\mathbf{R}^{n}, \rho\right)$ is defined to be $\max \left\{\right.$ form weight of $\left.\omega=\varepsilon_{i_{1}} \wedge \cdots \wedge \varepsilon_{i_{k}} \mid 1 \leqslant k \leqslant n\right\}$.

EXAMPLE. To illustrate these ideas we shall compute the invariant 1 -forms and the maximum form weight of the unipotent group using the canonical basis $\left\{u_{i j}\right.$ : $1 \leqslant i<j \leqslant n$ \} given in the example of 1.5 .

From 2.1 we have a basis for the invariant 1 -forms of $U_{n}(\mathbf{R})$ given at $x$ by $\varepsilon_{i, j}=r_{x^{-1}}^{*}\left(d x_{i j}\right)$. Hence by linearity of matrix multiplication $\varepsilon_{i, j}=\Sigma_{k}\left(x^{-1}\right)_{k, j} d x_{i, k}$. By an easy computation,

$$
\left(x^{-1}\right)_{i, j}=\sum_{r}(-1)^{r} x_{i_{1_{1}}} \cdot x_{i_{2} j_{2}} \cdots x_{i_{r} j_{r}}
$$

where the sum is taken over all pairs $\left(i_{n}, j_{n}\right)$ such that $i_{1}=i, j_{r}=j, j_{u}=i_{u+1}$, $i_{u}<j_{u}$.

PROPOSITION. The maximum form weight of $U_{n}(\mathbf{R})$ is $n(n-1) / 2$.

Proof. Let $G_{n}=U_{n}(\mathbf{R})$. Then the proof proceeds by induction on $n$, the result being clear for $n=1$. Consider the semidirect product

$$
0 \rightarrow \mathbf{R}^{n} \rightarrow G_{n+1} \stackrel{\pi}{\rightarrow} G_{n} \rightarrow 1
$$

where $\pi$ is projection on the first $n$ rows and columns. It follows that

$$
\Lambda_{G_{n+1}}^{*}\left(G_{n+1}\right) \approx \Lambda_{G_{n}}^{*}\left(G_{n}\right) \otimes \Lambda\left(\varepsilon_{1, n+1}, \ldots, \varepsilon_{n, n+1}\right) .
$$


Thus assuming the result for $n$, we are reduced to proving that the maximum form weight of $\Lambda\left(\varepsilon_{1, n+1}, \ldots, \varepsilon_{n, n+1}\right)$ is $n$. Now the total form degree of $\omega=\varepsilon_{i_{1}, n+1}$ $\wedge \cdots \wedge \varepsilon_{i_{k}, n+1}$ is $k$ plus the largest polynomial degree (in the $x_{i, j}$ 's) of the $k$-minors of $x^{-1}$ [MB, Lemma, p. 564]. However, if $\operatorname{det} A$ is a $k$-minor of $x^{-1}$, then $\operatorname{det} A=\operatorname{det} B$ where $B$ is the $n-k$ square matrix consisting of the rows (resp. columns) of $x$ complementary to the columns (resp. rows) of $x^{-1}$ which form $\operatorname{det} A$. This follows from the classical Jacobian Identities [G, p.191]. Thus the maximum form weight of $\omega$ is $k+n-k=n$.

3. Form cohomology. Let $\mathbf{Q}_{r}=\mathbf{Z}\left[\frac{1}{2}, \frac{1}{3}, \frac{1}{4}, \ldots, \frac{1}{r}\right]$. We shall use results of Cartan [C] to compute $H^{*}\left(X ; \mathbf{Q}_{r}\right)$ from a complex of "forms". Let $\Gamma\left(t_{0}, \ldots, t_{p}\right)$ denote the divided power algebra over $\mathbf{Z}$ and let

$$
B(p)=\Gamma\left(t_{0}, \ldots, t_{p}\right) \otimes \Lambda\left(d t_{0}, \ldots, d t_{p}\right) /\left(\sum d t_{i}\right)
$$

be the DGA with $d \gamma_{k}\left(t_{i}\right)=\gamma_{k-1}\left(t_{i}\right) d t_{i}, d\left(d t_{i}\right)=0$ and grading $\left|t_{i}\right|=0,\left|d t_{i}\right|=1$. The submodule of grading $q$ will be denoted by $B(p)^{q}$. Note $B(p)^{q}=0$ if $p<q$. Letting $t=\Sigma t_{i}$ we have an isomorphism

$$
B(p) \approx \Gamma(t) \otimes \Gamma\left(t_{1}, \ldots, t_{p}\right) \otimes \Lambda\left(d t_{i}, \ldots, d t_{p}\right)
$$

where $d t=0$ is understood. Since $\Gamma\left(t_{1}, \ldots, t_{p}\right) \otimes \Lambda\left(d t_{1}, \ldots, d t_{p}\right)$ is the classical Koszul complex, it is acyclic on $\mathbf{Z}$ [CE]. Thus $B(p)$ is acyclic on $\Gamma(t)$, i.e., $H^{0} B(p)=\Gamma(t), H^{q} B(p)=0, q>0$.

Elements $\omega\left(t_{0}, \ldots, t_{p}\right) \in B(p)$ may be considered as differential forms on the standard $p$-simplex $\Delta_{p}$ in the obvious way. ( $\Delta_{p}$ is the convex hull of $e_{1}, e_{2}, \ldots, e_{p+1}$.)

$B(*)$ can be given the structure of a simplicial DGA with face and degeneracy DGA maps

$$
\begin{gathered}
\partial_{i} \omega\left(t_{0}, \ldots, t_{p}\right)=\omega\left(t_{0}, \ldots, t_{i-1}, 0, t_{i}, \ldots, t_{p-1}\right), \\
s_{i} \omega\left(t_{0}, \ldots, t_{p}\right)=\omega\left(t_{0}, \ldots, t_{i-1}, t_{i}+t_{i+1}, t_{i+2}, \ldots, t_{p+1}\right)
\end{gathered}
$$

for $0 \leqslant i \leqslant p$.

$B(p)$ admits another grading; the form weight $s$ of $\gamma_{i_{0}}(t) \gamma_{i_{1}}\left(t_{1}\right) \cdots \gamma_{i_{p}}\left(t_{p}\right) d t_{j_{1}} \cdots d t_{j_{1}}$ is defined to be $s=\sum_{u=0}^{p} i_{u}+l$. This notion is compatible with the form weight of 2.3. Then $B(p)=\bigoplus_{s \geqslant 0} B(p)_{s}$ where $B(p)_{s}$ is the DG Z-module spanned by all monomials of form weight $s$. Furthermore, $B(*)=\bigoplus_{s \geqslant 0} B(*)_{s}$ is a direct sum of simplicial submodules.

Let $B(p, r)=\bigoplus_{s=0}^{r} B(p)_{s}$ then $B(p, r)$ is a DG Z-module acyclic on $\Gamma(t, r)=$ $\bigoplus_{s=0}^{r} \Gamma_{s}(t)$ where $\Gamma_{s}(t) \approx \mathbf{Z}$ is generated by $\gamma_{s}(t)$. Now let

$$
\mathbf{Q}_{r}(p)=[B(p, r) /(1-t) B(p, r-1)] \otimes \mathbf{Q}_{r} .
$$

If $\gamma_{s}(t) \in \mathbf{Q}_{r}(p)$ then $s \leqslant r$ hence $\gamma_{s}(t)=1 / s$ ! $\in \mathbf{Q}_{r}$. Thus a $\mathbf{Q}_{r}$ basis for $\mathbf{Q}_{r}(p)$ consists of monomials $\gamma_{i_{1}}\left(t_{1}\right) \cdots \gamma_{i_{p}}\left(t_{p}\right) d t_{j_{1}} \cdots d t_{j_{1}}$ of total form weight $s \leqslant r$ and $j_{u} \geqslant 1$. Clearly $\mathbf{Q}_{r}(*)$ inherits the structure of a simplicial DG $\mathbf{Q}_{r}$-module.

3.1 Definition. Let $X$ be a simplicial set and define the DG $\mathbf{Q}_{r}$-module $\mathbf{Q}_{r}(X)$ by

$$
\mathbf{Q}_{r}^{q}(X)=\mathfrak{S}\left(X, \mathbf{Q}_{r}(*)^{q}\right)
$$

where $\delta(\cdot, \cdot)$ denotes maps of simplicial sets. 
3.2 THEOREM. There is a natural isomorphism

$$
\begin{aligned}
H^{k}\left(\mathbf{Q}_{r}(X)\right) & \approx H^{k}\left(X ; \mathbf{Q}_{r}\right), & & k \leqslant r, \\
& =0, & & k>r .
\end{aligned}
$$

Proof. The proof consists of showing that $\mathbf{Q}_{r}(*)$ satisfies Cartan's Axioms (a) and $\left(b^{\prime}\right)$. The result then follows from Cartan $[\mathbf{C}, \S 6]$.

Let $Z^{q} \mathbf{Q}_{r}(p)=\operatorname{ker} d: \mathbf{Q}_{r}(p)^{q} \rightarrow \mathbf{Q}_{r}(p)^{q+1}$.

3.3 Lemma (Aхіом (a)). $\mathbf{Q}_{r}(p)^{*}$ is acyclic on $\mathbf{Q}_{r}$ and $Z^{0} \mathbf{Q}_{r}(*)$ is simplicially trivial (i.e. all $\left.\partial_{i}=s_{i}=\mathrm{id}\right)$.

Proof. Since $B(p, r)$ is acyclic on $\Gamma(0, r)$ and since localization preserves exactness, the first result follows from the long exact sequence in cohomology induced by the exact sequence of DG modules

$$
0 \rightarrow B(p, r-1) \otimes \mathbf{Q}_{r} \stackrel{\times(1-t)}{\rightarrow} B(p, r) \otimes \mathbf{Q}_{r} \rightarrow \mathbf{Q}_{r}(p) \rightarrow 0 .
$$

The second result is clear since $Z^{0} B(*, r)=\Gamma(t, r)$ is simplicially trivial.

3.4 LeMMA (AхIOM $\left(\mathrm{b}^{\prime}\right)$ ). $\pi_{k} \mathbf{Q}_{r}(*)^{q}$ and $\pi_{k} Z^{q} \mathbf{Q}_{r}(*)$ are zero for $k \neq q$ and $\pi_{k} Z^{q} \mathbf{Q}_{r}(*) \rightarrow \pi_{k} \mathbf{Q}_{r}(*)^{q}$ is surjective with the kernel a direct summand as $\mathbf{Q}_{r}$-modules.

Proof. First we compute $\pi_{k} \mathbf{Q}_{r}(*)^{q}$ and $\pi_{k} Z^{q} \mathbf{Q}_{r}(*)$. Cartan $[\mathbf{C}$, p. 270] has shown

$$
\begin{gathered}
\pi_{k} B(*)^{q} \approx \mathbf{Z}, \quad k=q, \\
\pi_{k} Z^{q} B(*)^{q} \approx \bigoplus_{s \geqslant q} \Gamma_{s}(t), \quad k=q,
\end{gathered}
$$

with the remaining groups being zero for $k \neq q$. In (2), $d t_{1} \cdots d t_{q}$ is a generator ( 1 is a generator for $q=0$ ) and multiplication by $d t_{q}$ is an isomorphism

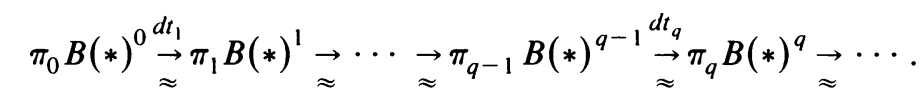

In (3), $\gamma_{s-q}(t) d t_{1} \cdots d t_{q}$ is the generator corresponding to $\gamma_{s}(t)$. Thus from the definition of $B(*, r)$ we have

$$
\begin{aligned}
\pi_{k} B(*, r)^{q} & \approx \mathbf{Z}, \quad k=q \leqslant r, \\
& =0, \quad k \neq q \text { or } q>r, \\
\pi_{k} Z^{q} B(*, r)^{q} & \approx \bigoplus_{s=q}^{r} \Gamma_{s}(t), \quad k=q \leqslant r, \\
& =0, \quad k \neq q \text { or } q>r,
\end{aligned}
$$

(in fact $B(*, r)^{q}=0$ if $q>r$ ).

For fixed $q$ and variable $p,(1)$ is a Kan fibration since it is a short exact sequence of simplicial groups. Further $t=0$ in $\pi_{0} B(*, r)$ and multiplication by $t$ commutes with multiplication by $d t_{q}$ in (4). Thus from (1) and (5) we have

$$
\begin{aligned}
\pi_{k} \mathbf{Q}_{r}(*)^{q} & \approx \mathbf{Q}_{r}, \quad k=q=r \\
& =0, \quad \text { otherwise. }
\end{aligned}
$$


Since $B(p, r)$ is acyclic, it follows from (1) that

$$
0 \rightarrow Z^{q} B(*, r-1) \otimes \mathbf{Q}_{r} \stackrel{\times(1-t)}{\rightarrow} Z^{q} B(*, r) \otimes \mathbf{Q}_{r} \rightarrow Z^{q} \mathbf{Q}_{r}(*) \rightarrow 0
$$

is exact. Furthermore it is easy to check that the correspondence $\gamma_{s-q}\left(t_{q}\right) d t_{1} \cdots d t_{q}$ $\mapsto \gamma_{s}(t)$ commutes with multiplication by $t$, hence by (1) and (6) we have

$$
\begin{aligned}
\pi_{k} Z^{q} \mathbf{Q}_{r}(*) & =\mathbf{Q}_{r}, & & k=q \leqslant r, \\
& =0, & & \text { otherwise. }
\end{aligned}
$$

Finally the map $\pi_{k} Z^{q} \mathbf{Q}_{r}(*) \rightarrow \pi_{k} \mathbf{Q}_{r}(*)^{q}$ is clearly onto with kernel a direct summand since the only nonzero case is $k=q=r$ where an isomorphism holds. This completes the proof of Lemma 3.4 and hence of the theorem.

If $Y \subset X$ is a subsimplicial set, let $\mathbf{Q}_{r}^{*}(X, Y)$ be the kernel of $\mathbf{Q}_{r}^{*}(Y) \rightarrow \mathbf{Q}_{r}^{*}(X)$. Then $\mathbf{Q}_{r}^{*}(X, Y)$ is a DG $\mathbf{Q}_{r}$-module. Furthermore,

3.5 Proposition. If $Y \subset X$ and $\operatorname{dim} X \leqslant r$ then

$$
0 \rightarrow \mathbf{Q}_{r}^{*}(X, Y) \rightarrow \mathbf{Q}_{r}^{*}(X) \rightarrow \mathbf{Q}_{r}^{*}(Y) \rightarrow 0
$$

is exact.

Proof. Except at $\mathbf{Q}_{r}^{*}(Y)$, exactness is clear by definition. By (7), $\pi_{*} \mathbf{Q}_{r}(*)^{q}=0$ unless $q=r$ in which case $\mathbf{Q}_{r}(*)^{r}=K\left(\mathbf{Q}_{r}, r\right)$. If $f: Y \rightarrow K\left(\mathbf{Q}_{r}, r\right)$ is a map of simplicial sets then $f$ extends over $X$ by ordinary obstruction theory since $\operatorname{dim} X \leqslant r$ implies $H^{r+1}\left(X, Y ; \mathbf{Q}_{r}\right)=0$.

3.6 Remark. Let $r \rightarrow \infty$ and set $\mathbf{Q}_{\infty}=\mathbf{Q}$. Then $\mathbf{Q}_{\infty}^{*}(X)$ is Cartan's simplicial version of Sullivan's rational de Rham theory [C, p. 265]. In this case $H^{*}(\mathbf{Q}(X)) \approx$ $H^{*}(X ; \mathbf{Q})$ as rings.

4. The main theorem. Let $B D$ be the simplicial classifying complex of $D$. Our goal is to construct a map of DG modules

$$
\kappa: \Lambda^{*}\left(G ; \mathbf{Q}_{r}\right) \rightarrow \mathbf{Q}_{r}^{*}(B D)
$$

inducing an isomorphism in cohomology. Here $r$ is chosen so that $\mathbf{Q}_{r} \supset R$, the subring of $\mathbf{Q}$ generated by the coefficients of the multiplication $\rho$ (see 1.8).

We shall use the simplicial model for $B D$ defined by $B D_{0}=0, B D_{p}=D^{p}$ with

$$
\begin{aligned}
& \partial_{i}\left(d_{p}, \ldots, d_{1}\right)= \begin{cases}\left(d_{p}, \ldots, d_{2}\right), & i=0, \\
\left(d_{p}, \ldots, d_{i+1} d_{i}, \ldots, d_{1}\right), & 0<i<p, \\
\left(d_{p-1}, \ldots, d_{1}\right), & i=p,\end{cases} \\
& s_{i}\left(d_{p}, \ldots, d_{1}\right)= \begin{cases}\left(d_{p}, \ldots, d_{1}, 0\right), & i=0, \\
\left(d_{p}, \ldots, d_{i+1}, 0, d_{i}, \ldots, d_{1}\right), & 0<i<p, \\
\left(0, d_{p}, \ldots, d_{1}\right), & i=p .\end{cases}
\end{aligned}
$$

To relate $B D$ and $G / D$ we construct a family of compatible maps $\left\{g_{(d) \text { : }}\right.$ $\left.\Delta_{p} \rightarrow G / D\right\}$ indexed by $d=\left(d_{p}, \ldots, d_{1}\right) \in B D_{p}$. For $s \in \mathbf{R}$ let $s d=\left(s d_{p}, \ldots, s d_{1}\right) \in$ $G$ and define

$$
\mathfrak{s}\left(d_{p}, \ldots, d_{1}\right)\left(t_{0}, \ldots, t_{p}\right)=\mathfrak{s}_{1}\left(\cdots \mathfrak{g}_{p-1}\left(\left(\mathfrak{\xi}_{p} d_{p}\right) \cdot d_{p-1}\right) \cdots d_{1}\right)
$$


where $\mathfrak{S}_{1}, \ldots, \mathfrak{S}_{p}$ are the cone coordinates

$$
\mathfrak{s}_{k}=\left(1-t_{0}-\cdots-t_{k-1}\right) /\left(1-t_{0}-\cdots-t_{k-2}\right) .
$$

Similar maps have been defined, e.g. [ST]. Our map $s$ is chosen so as to minimize form weight.

The multiplication $\rho$ is said to be semilinear if $\rho_{i}(x, y)=x_{i}+y_{i}+\sum_{j} x_{j} p_{i j}(y)$ where $p_{i j}(y)$ is a polynomial in the variables $y_{1}, \ldots, y_{i-1}$. In particular, this includes those $\rho$ with terms of degree at most two.

All of the examples considered in this paper are semilinear and it is important to note that in this case $\mathfrak{Z}(d)(t)$ is a linear function of the $t_{i}$.

Initially we define $\kappa: \Lambda^{*}(G ; \mathbf{Q}) \rightarrow \mathbf{Q}_{\infty}^{*}(B D)$ over $\mathbf{Q}$ by $\kappa(\omega)(d)=\mathfrak{s}(d)^{*}(\omega)$, the pullback of $\omega$ to $\Delta_{p}$. It is straightforward to check that $\kappa(\omega): B D \rightarrow \mathbf{Q}(*)$ is a simplicial map and hence an element of $\mathbf{Q}_{\infty}^{*}(B D)$. Furthermore since pullbacks commute with differentials and products, $\kappa d=d \kappa$ and $\kappa\left(\omega \omega^{\prime}\right)=\kappa(\omega) \kappa\left(\omega^{\prime}\right)$.

To state our main result we assume $\rho$ is semilinear. We also assume $r \geqslant \sum r_{i}$ where $r_{i}$ is the form weight of $\varepsilon_{i}$, the basic invariant 1-forms of $G$ (see 2.3).

4.1 THEOREM. $\kappa$ restricts to a DG module map $\kappa: \Lambda^{*}\left(G ; \mathbf{Q}_{r}\right) \rightarrow \mathbf{Q}_{r}^{*}(B D)$ which induces an isomorphism in cohomology. Hence

$$
H^{*}\left(L_{\mathbf{Q}_{r}}(D) ; \mathbf{Q}_{r}\right) \approx H^{*}\left(B D ; \mathbf{Q}_{r}\right) \text {. }
$$

The rational version of Nomizu's theorem [N], [Su, Theorem 8.1] is

4.2 COROLlaRY. Let $r=\infty$. Then $\kappa$ induces an isomorphism of cohomology rings over $\mathbf{Q}$.

Proof of Theorem 4.1. $\kappa$ restricts as stated since the maximum form weight of $G$ is $\leqslant r$ and $\mathfrak{s}(d)^{*}$ is linear. Now using the refinement (1.3) of the upper central series of $D$

$$
D=D_{1} \supset D_{2} \supset \cdots \supset D_{n} \supset D_{n+1}=1,
$$

we can build up $D$ by a series of central extensions

$$
0 \rightarrow \mathbf{Z} \rightarrow D(k) \stackrel{\pi}{\rightarrow} D(k-1) \rightarrow 1
$$

where $D(k)=D / D_{k+1}$ so that $D(1)=\mathbf{Z}, D(n)=D$. Let $G(k)=D_{\mathbf{R}}(k)$ be the real completion of $D(k)$ and set $s_{k}=r_{1}+\cdots+r_{k}$. Since $B D(1) \simeq S^{1}$, the theorem is easily seen to be true for $D(1)$ with $\mathbf{Q}_{r}^{*}(\cdot)$ replaced by $\mathbf{Q}_{s_{1}}(\cdot) \otimes \mathbf{Q}_{r}$. For $k-1$, we assume

$$
\kappa: \Lambda^{*}\left(G(k-1) ; \mathbf{Q}_{r}\right) \rightarrow \mathbf{Q}_{s_{k-1}}(B D(k-1)) \otimes \mathbf{Q}_{r}
$$

induces an isomorphism in cohomology. Upon completion, (1) yields

$$
\mathbf{R} \rightarrow G(k) \stackrel{\pi}{\rightarrow} G(k-1)
$$

and by $2.1, d \varepsilon_{k}=\pi^{*}(\omega)$ where $\omega$ is a $G(k-1)$ invariant 2-form. Now consider the chain complex $\left[\mathbf{Q}_{s_{k-1}}^{*}(B D(k-1)) \otimes \mathbf{Q}_{r}\right] \otimes_{d} \Lambda\left(\varepsilon_{k}\right)$ with $d \varepsilon_{k}=\kappa(\omega)$. We shall consider this complex as associated with the principal bundle

$$
S^{1} \simeq B \mathbf{Z} \stackrel{i}{\rightarrow} B D(k) \stackrel{B \pi}{\rightarrow} B D(k-1)
$$


induced by (1). To define the desired equivalence let $\rho:\left[\mathbf{Q}_{s_{k-1}}^{*}(B D(k-1)) \otimes \mathbf{Q}_{r}\right]$ $\otimes_{d} \Lambda\left(\varepsilon_{k}\right) \rightarrow \mathbf{Q}_{r}^{*}(B D(k))$ be the composite

$$
\begin{aligned}
& {\left[\mathbf{Q}_{s_{k-1}}^{*}(B D(k-1)) \otimes \mathbf{Q}_{r}\right] \otimes_{d} \Lambda\left(\varepsilon_{k}\right) } \\
& \quad \stackrel{B \pi^{*} \otimes \kappa}{\rightarrow}\left[\mathbf{Q}_{s_{k-1}}^{*}(B D(k)) \otimes \mathbf{Q}_{r}\right] \otimes\left[\mathbf{Q}_{r_{k}}^{*}(B D(k)) \otimes \mathbf{Q}_{r}\right] \stackrel{\Lambda}{\rightarrow} \mathbf{Q}_{r}^{*}(B D(k))
\end{aligned}
$$

where $\Lambda$ is induced by the product of forms.

4.3 LeMMA (HIRSCH LEMMA). $\rho$ induces an isomorphism in cohomology.

Proof. The proof proceeds by induction on the cells of the base in (3). Assume the lemma for $B \subset B D(n-1)$. Since $\rho$ is natural we have

$$
\begin{aligned}
0 \leftarrow\left[\mathbf{Q}_{s_{n-1}}^{*}(B) \otimes \mathbf{Q}_{r}\right] \otimes_{d} \Lambda\left(\varepsilon_{n}\right) & \leftarrow\left[\mathbf{Q}_{s_{n-1}}^{*}\left(\Delta_{k} \cup B\right) \otimes \mathbf{Q}_{r}\right] \otimes_{d} \Lambda\left(\varepsilon_{n}\right) \\
\downarrow \leftarrow \rho & \leftarrow \quad \begin{array}{l}
\downarrow \rho \\
\mathbf{Q}_{r}^{*}\left(B \pi^{-1}(B)\right)
\end{array} \\
& \leftarrow\left[\mathbf{Q}_{r}^{*}\left(B \pi^{-1}\left(\Delta_{k} \cup B\right)\right)\right. \\
& \left.\leftarrow \quad \mathbf{Q}_{r}^{*-1}\left(\Delta_{k} \cup B, B\right) \otimes \mathbf{Q}_{r}\right] \otimes_{d} \Lambda\left(\varepsilon_{n}\right) \leftarrow 0
\end{aligned}
$$

Now $B \pi^{-1}\left(\Delta_{k} \cup B, B\right) \simeq\left(\Delta_{k} \cup B, B\right) \times S^{1}$ and $i^{*}\left(\kappa\left(\varepsilon_{n}\right)\right)$ is a closed 1 -form which represents a generator of $H^{1}\left(\mathbf{Q}_{s}(B \mathbf{Z}) \otimes \mathbf{Q}_{r}\right) \approx \mathbf{Q}_{r}$ for $s \geqslant 1$. The result follows from the Künneth Theorem and the five lemma.

To complete the proof of the theorem, we note that

$$
\Lambda^{*}\left(G(n) ; \mathbf{Q}_{r}\right)=\Lambda^{*}\left(G(n-1) ; \mathbf{Q}_{r}\right) \otimes_{d} \Lambda\left(\varepsilon_{n}\right) \quad \text { where } d \varepsilon_{n} \in \Lambda^{*}\left(G(n-1) ; \mathbf{Q}_{r}\right) \text {. }
$$

Thus $\kappa$ is the composite

$$
\Lambda^{*}\left(G(n-1) ; \mathbf{Q}_{r}\right) \otimes_{d} \Lambda\left(\varepsilon_{n}\right) \stackrel{\kappa \otimes 1}{\rightarrow}\left[\mathbf{Q}_{s_{n-1}}^{*}(B D(n-1)) \otimes \mathbf{Q}_{r}\right] \otimes_{d} \Lambda\left(\varepsilon_{n}\right) \stackrel{\rho}{\rightarrow} \mathbf{Q}_{r}(B D(n))
$$

and $\kappa \otimes 1$ induces an isomorphism in cohomology by (2) and the comparison theorem for spectral sequences [Mc, Chapter XI, Theorem 11.1].

5. A naturality result. In this section, we show how a choice of canonical bases for finitely generated, torsion-free, nilpotent groups $D$ and $E$ gives rise to an explicit description of any homomorphism $f: D \rightarrow E$ as a polynomial function which may be used to describe the various localizations of $f$. We also classify the homomorphisms from $D$ to $E$ in terms of Lie algebra maps and use this to show a certain naturality in the isomorphism 4.1.

5.1 Explicit description of a localization functor. Suppose $D \approx\left(\mathbf{Z}^{n}, \rho\right)$ and $E \approx$ $\left(\mathbf{Z}^{m}, \eta\right)$ with respect to canonical bases $\left\{u_{1}, \ldots, u_{n}\right\},\left\{v_{1}, \ldots, v_{m}\right\}$ as in (1.6).

If $f: D \rightarrow E$ is a homomorphism then

$$
f\left(u_{1}^{x_{1}} \cdots u_{n}^{x_{n}}\right)=f\left(u_{1}\right)^{x_{1}} \cdots f\left(u_{n}\right)^{x_{n}}
$$


so that $f$ is uniquely determined by $f\left(u_{j}\right)=v_{1}^{f_{1 j}} \cdots v_{m}^{f_{m j}}, f_{i j} \in \mathbf{Z}$. If $p_{k}: E \rightarrow E$ denotes the $k$ th power map $p_{k}(x)=x^{k}$ then by using $\left\{v_{1}, \ldots, v_{m}\right\}$ we have that $p_{k}$ : $\mathbf{Z}^{m} \rightarrow \mathbf{Z}^{m}$ is a polynomial function with $p_{k}(x)=k x+$ terms of degree $\geqslant 2$ [S, LG 4.19]. Also (1.5) gives $\eta(x, y)=x+y+$ terms of degree $\geqslant 2$.

Combining these facts we see that $f: \mathbf{Z}^{n} \rightarrow \mathbf{Z}^{m}$ is represented as a polynomial function $f(x)=\left(f_{1}(x) \cdots f_{m}(x)\right)$ where $f_{j}(x)=\sum_{i=1}^{n} f_{i j} x_{j}+$ terms of degree $\geqslant 2$.

Thus if $R$ is the subring of $\mathbf{Q}$ generated by the coefficients of $\rho$ or $\eta$ and $\tilde{R}$ is any ring such that $R \subset \tilde{R} \subset \mathbf{Q}$ or $\tilde{R}=\mathbf{R}$, then we have the induced homomorphism $f_{\tilde{R}}$ : $D_{\tilde{R}} \rightarrow E_{\tilde{R}}$ where $f_{\tilde{R}}$ is the polynomial function given by the same polynomial as $f$ (in the choice of canonical bases). When $\tilde{R}=\mathbf{R}, f_{\mathbf{R}}$ is also a Lie group map. This construction is functorial and when $\tilde{R} \subset \mathbf{Q}$, it gives a description of the usual localization functor.

5.2 The induced Lie algebra map. Let $D, E, \rho, \eta, f, \tilde{R}$ be as in 5.1 . We have the algebraic Lie algebras $L_{\tilde{R}}(D)$ and $L_{\tilde{R}}(E)$ as in 1.8 and we have the functor $L$ which assigns a Lie algebra map $L(f): L_{\tilde{R}}(D) \rightarrow L_{\tilde{R}}(E)$ to any group homomorphism $f$ : $D_{\tilde{R}} \rightarrow E_{\tilde{R}}$ where $L(f)=$ linear part of the polynomial function $f$.

5.3 Proposition. $L$ is a bijection from the set of group homomorphisms $D_{\tilde{R}} \rightarrow E_{\tilde{R}}$ to the set of Lie algebra homomorphisms $L_{\tilde{R}}(D) \rightarrow L_{\tilde{R}}(E)$.

Proof. It is enough to show that if $\phi: L_{\tilde{R}}(D) \rightarrow L_{\tilde{R}}(E)$ is a Lie algebra map, then there is a unique group homomorphism $f$ such that $L(f)=\phi$. Thus suppose $\phi\left(e_{j}\right)=\sum_{i=1}^{m} \phi_{i j} e_{i}$ where $e_{i}, e_{j}$ denote the standard basis vectors. Define a function $f:$ $D_{\tilde{R}} \rightarrow E_{\tilde{R}}$ by setting $f\left(u_{j}\right)=v_{1}^{\phi_{1 j}} v_{2}^{\phi_{2} j} \cdots v_{m}^{\phi_{m j}}$ and then

$$
f\left(u_{1}^{x_{1}} u_{2}^{x_{2}} \cdots u_{n}^{x_{n}}\right)=f\left(u_{1}\right)^{x_{1}} f\left(u_{2}\right)^{x_{2}} \cdots f\left(u_{n}\right)^{x_{n}} .
$$

We are done if $f$ is a homomorphism since $f$ is a polynomial function with linear part $\phi$; but we have a smooth map of manifolds $\hat{f}: D_{\mathbf{R}} \rightarrow E_{\mathbf{R}}$ given by the same polynomial that gives $f$ and since these manifolds are just Euclidean spaces, the derivative of $\hat{f}$ at the identity is just the linear part of $\hat{f}$, which is just $\phi \otimes \mathbf{R}$. But since $\phi \otimes \mathbf{R}$ is obviously a Lie algebra map, $\hat{f}$ must be a Lie group homomorphism $\left[\mathbf{V}\right.$, p. 171] and hence $f=\left.\hat{f}\right|_{D_{\tilde{\mathbf{R}}}}$ is a homomorphism.

5.4 COROLlaRY. If $\rho$ and $\eta$ have integer coefficients, then

$$
[D, E]_{\mathrm{Gp}} \stackrel{L}{\rightarrow}\left[L_{\mathbf{Z}}(D), L_{\mathbf{Z}}(E)\right]_{\text {Lie alg }}
$$

is a bijection.

5.5 Naturality. Let $f: M \rightarrow N$ be a map of nilmanifolds. Then the homomorphism $f_{*}: \pi_{1} M=D \rightarrow E=\pi_{1} N$ induces a map $g=f_{* \mathbf{R}} / f_{*}: M=D_{\mathbf{R}} / D \rightarrow E_{\mathbf{R}} / E=N$. Since $M$ and $N$ are $K(\pi, 1)$ spaces, $f \simeq g$. Thus for cohomological considerations we may assume $f=g$ and then by $L(f)$ we mean $L\left(f_{* \mathbf{Q}_{s}}\right)$ of 5.2 where $\mathbf{Q}_{s}$ is the subring of $\mathbf{Q}$ generated by the union of the rings corresponding to $M$ and $N$ as in Theoem 4.1. 
Proposition. If $f: M \rightarrow N$ is a map of nilmanifolds, then under the assumptions of Theorem 4.1

$$
\begin{array}{ccc}
H^{*}\left(L_{\mathbf{Q}_{s}}(M)\right) & \stackrel{L(f)^{*}}{\leftarrow} & H^{*}\left(L_{\mathbf{Q}_{s}}(N)\right) \\
\kappa \downarrow \approx & & \kappa \downarrow \approx \\
H^{*}\left(M ; \mathbf{Q}_{s}\right) & \stackrel{f^{*}}{\leftarrow} & H^{*}\left(N ; \mathbf{Q}_{s}\right)
\end{array}
$$

commutes.

Proof. The naturality of $\kappa$ implies the commutativity of

$$
\begin{array}{ccc}
\Lambda^{*}\left(D_{\mathbf{R}} ; \mathbf{Q}_{s}\right) & \leftarrow & \Lambda^{*}\left(E_{\mathbf{R}} ; \mathbf{Q}_{s}\right) \\
\downarrow \kappa & & \downarrow_{\kappa} \\
\mathbf{Q}_{s}^{*}(B D) & \leftarrow & \mathbf{Q}_{s}^{*}(B E)
\end{array}
$$

The proposition follows using the isomorphism $\Lambda^{*}\left(D_{\mathbf{R}} ; \mathbf{Q}_{s}\right) \approx \Lambda^{*}\left(L_{\mathbf{Q}_{s}}(D)^{*}\right)$ of 2.2.

6. Calculations.

6.1 Generalized Heisenberg group. Let $k$ be a fix integer and let

$$
U_{3}(k)=\left\{\left(\begin{array}{lll}
1 & x_{12} & x_{13} / k \\
0 & 1 & x_{23} \\
0 & 0 & 1
\end{array}\right): x_{i, j} \in \mathbf{Z}\right\}
$$

Then $U_{3}(k)$ is a torsion-free, nilpotent group with a canonical basis given by

$$
u_{12}=\left(\begin{array}{lll}
1 & 1 & 0 \\
0 & 1 & 0 \\
0 & 0 & 1
\end{array}\right), \quad u_{23}=\left(\begin{array}{ccc}
1 & 0 & 0 \\
0 & 1 & 1 \\
0 & 0 & 1
\end{array}\right), \quad u_{13}=\left(\begin{array}{ccc}
1 & 0 & 1 / k \\
0 & 1 & 0 \\
0 & 0 & 1
\end{array}\right)
$$

Thus $U_{3}(k) \approx\left(\mathbf{Z}^{3}, \rho\right)$ with

$$
\rho_{12}(x, y)=x_{2}+y_{12}, \quad \rho_{23}(x, y)=x_{23}+y_{23}, \quad \rho_{13}(x, y)=x_{13}+y_{13}+k x_{12} y_{23} \text {. }
$$

The algebraic group Lie algebra $L\left(U_{3}(k)\right)$ has as Z-basis $\left\{u_{12}, u_{23}, u_{13}\right\}$ with $\left[u_{12}, u_{23}\right]=k u_{13}$. In this case, a simple computation shows that as rings

$$
H^{*}\left(U_{3}(k) ; \mathbf{Z}\right) \approx H^{*}\left(L U_{3}(k) ; \mathbf{Z}\right)=\Lambda\left[v_{12}, v_{23}, v_{12} v_{13}, v_{23} v_{13}\right] /\left(k v_{12} v_{23}\right)
$$

where $v_{i j}$ corresponds to $u_{i j}$ in the dual basis.

6.2 Generalized unipotent group. Let $k, l$ be fixed integers and let

$$
D=U_{4}(k, l)=\left\{\left(\begin{array}{cccc}
1 & x_{12} & x_{13} / k & x_{14} / k l \\
0 & 1 & x_{23} & x_{24} / l \\
0 & 0 & 1 & x_{34} \\
0 & 0 & 0 & 1
\end{array}\right): x_{i j} \in \mathbf{Z}\right\}
$$

Then $D$ is a torsion-free, nilpotent group of rank 6. A canonical basis is given by $\left\{u_{i j}: 1 \leqslant i<j \leqslant 4\right\}$ where $u_{i j}$ is the matrix in $D$ with $x_{i j}=1$ and $x_{u v}=0, u v \neq i j$. 
In these coordinates $D \approx\left(\mathbf{Z}^{6}, \rho\right)$ where

$$
\rho_{i j}(x, y)= \begin{cases}x_{i j}+y_{i j} & i j=12,23,34, \\ x_{13}+y_{13}+k x_{12} y_{23} & i j=13, \\ x_{24}+y_{24}+l x_{23} y_{34} & i j=24, \\ x_{14}+y_{14}+k x_{12} y_{24}+l x_{13} y_{34} & i j=14 .\end{cases}
$$

There are central extensions

$$
0 \rightarrow \mathbf{Z}\left\langle u_{14}\right\rangle \rightarrow D \rightarrow D^{\prime} \rightarrow 1
$$

and

$$
0 \rightarrow \mathbf{Z}\left\langle u_{13}, u_{24}\right\rangle \rightarrow D^{\prime} \rightarrow \mathbf{Z}\left\langle u_{12}, u_{23}, u_{34}\right\rangle \rightarrow 1
$$

where $D^{\prime}$ is defined by (1).

$L\left(D^{\prime}\right)$ has as Z-basis $\left\{u_{12}, u_{23}, u_{34}, u_{13}, u_{24}\right\}$ with $\left[u_{12}, u_{23}\right]=k u_{13},\left[u_{23}, u_{34}\right]=$ $l u_{24}$. The integral cohomology ring is given by

$$
\begin{aligned}
& H^{1} \approx \mathbf{Z}\left\langle v_{12}, v_{23}, v_{34}\right\rangle, \\
& H^{2} \approx \mathbf{Z}\left\langle v_{12} v_{13}, v_{12} v_{34}, v_{23} v_{13}, v_{23} v_{24}, v_{34} v_{24}, x v_{12} v_{24}+y v_{13} v_{34}\right\rangle \\
& \oplus \mathbf{Z} / k\left\langle v_{12} v_{23}\right\rangle \oplus \mathbf{Z} / l\left\langle v_{23} v_{34}\right\rangle, \\
& H^{3} \approx \mathbf{Z}\left\langle v_{12} v_{23} v_{13}, v_{12} v_{13} v_{34}, v_{12} v_{34} v_{24},\right. \\
& \left.v_{23} v_{13} v_{24}, v_{23} v_{34} v_{24}, s v_{12} v_{23} v_{24}-t v_{23} v_{13} v_{34}\right\rangle \\
& \oplus \mathbf{Z} / d\left\langle x v_{12} v_{23} v_{24}+y v_{23} v_{13} v_{34}\right\rangle \oplus \mathbf{Z} / d\left\langle x_{12} x_{23} x_{34}\right\rangle
\end{aligned}
$$

where $d=\operatorname{gcd}(k, l), x=k / d, y=l / d, x t+y s=1$. The remaining cohomology is determined by Poincaré duality.

The integral group cohomology $H^{*}\left(D^{\prime} ; \mathbf{Z}\right)$ can be computed by (2). However, if $d>1$, then the Serre spectral sequence has an extension problem in $\operatorname{dim} 3$ and a product in $\operatorname{dim} 4$ which is determined only up to filtration. This illustrates the problems encountered using extensions (1) and (2) to compute the cohomology of $D$ and $D^{\prime}$. Instead of pursuing this line, we shall apply our main result.

The invariant 1 -forms of $G^{\prime}=D_{\mathbf{R}}^{\prime}$ are $\varepsilon_{i j}=d x_{i j}, i j=12,23,34, \varepsilon_{13}=d x_{13}-$ $k x_{12} d x_{23}, \varepsilon_{24}=d x_{24}-l x_{23} d x_{34}$ (here for convenience we use left invariant forms). Thus by the theorem of $\S 4$

$$
H^{*}\left(D^{\prime} ; \mathbf{Q}_{r}\right) \approx H^{*}\left(L D^{\prime} ; \mathbf{Q}_{r}\right)
$$

if $r \geqslant 7$. (The sum of the form weights of the $\varepsilon_{i j}$ is 7 and the maximum form weight of $G^{\prime}$ is 5 .)

Similarly, $L D \approx L D^{\prime} \oplus \mathbf{Z}\left\langle u_{14}\right\rangle$ as modules with $\left[u_{12}, u_{24}\right]=k u_{14},\left[u_{13}, u_{34}\right]=$ $l u_{14}$. The additional invariant 1 -form is $\varepsilon_{14}=d x_{14}-k x_{12} d x_{24}-l x_{13} d x_{34}+$ $k l x_{12} x_{23} d x_{34}$. Thus if $r \geqslant 10, H^{*}\left(D ; \mathbf{Q}_{r}\right) \approx H^{*}\left(L D ; \mathbf{Q}_{r}\right)$ as modules.

The integral cohomology $H^{*}(L D ; \mathbf{Z})$ is given by

$$
\begin{aligned}
H^{1} \approx & \mathbf{Z}\left\langle v_{12}, v_{23}, v_{34}\right\rangle, \\
H^{2} \approx & \mathbf{Z}\left\langle v_{12} v_{13}, v_{12} v_{34}, v_{13} v_{23}, v_{23} v_{24}, v_{24} v_{34}\right\rangle \oplus \mathbf{Z} / k\left\langle v_{12} v_{23}\right\rangle \oplus \mathbf{Z} / l\left\langle v_{23} v_{34}\right\rangle \\
& \oplus \mathbf{Z} / d\left\langle x v_{12} v_{24}+y v_{13} v_{34}\right\rangle,
\end{aligned}
$$




$$
\begin{aligned}
H^{3} \approx & \mathbf{Z}\left\langle v_{12} v_{13} v_{14}, v_{12} v_{13} v_{23}, v_{12} v_{14} v_{34}, v_{13} v_{23} v_{24}, v_{14} v_{24} v_{34}, v_{23} v_{24} v_{34}\right\rangle \\
& \oplus \mathbf{Z} / k\left\langle v_{12} v_{24} v_{34}, v_{12} v_{13} v_{24} k-v_{12} v_{14} v_{23}\right\rangle \\
& \oplus \mathbf{Z} / l\left\langle v_{12} v_{13} v_{34}, v_{13} v_{24} v_{34}+v_{14} v_{23} v_{34}\right\rangle \\
& \oplus \mathbf{Z} / d\left\langle v_{12} v_{23} v_{34}, x v_{12} v_{23} v_{24}+y v_{13} v_{23} v_{34}\right\rangle \oplus \mathbf{Z} / 2 k y\left\langle s v_{12} v_{23} v_{24}-t v_{13} v_{23} v_{34}\right\rangle
\end{aligned}
$$

with $d=\operatorname{gcd}(k, l)$ as before. Once again the remaining cohomology is determined by Poincaré duality.

7. Alternate Lie algebras. In this paper, we have considered the algebraic group Lie algebra $L_{R}(D)$ associated with the torsion-free, nilpotent group $D$. There are, of course, other Lie algebras which can be associated with $D$. Most of these are grades. For example, Lazard [Lz] has observed that if $G$ is a group and $\left\{G_{i}\right\}$ is an $N$-sequence of subgroups, i.e. $G_{1}=G,\left(G_{i}, G_{j}\right) \subset G_{i+j}$ then $\bigoplus G_{i} / G_{i+1}$ is a graded Lie algebra with addition induced by the group product and bracket induced by the commutator. A classical example of an $N$-sequence is the lower central series $\left\{\Gamma_{i} G\right\}$ defined inductively by $\Gamma_{1} G=G / \Gamma_{i+1} G=\left(G, \Gamma_{i} G\right)$. The resulting Lie algebra $l(G)=$ $\bigoplus \Gamma_{i} G / \Gamma_{i+1} G$ has been studied extensively $[\mathbf{H}, \mathbf{Q}]$. From a homological point of view, part of this interest stems from the fact that $\left\{\Gamma_{i} G\right\}$ induces a filtration of the classifying complex $B G$ and hence a spectral sequence which takes the form $E_{2} \Rightarrow H^{*}(G ; \mathbf{Z})$ with $E_{2}=H^{*}(l(G) ; \mathbf{Z})$.

In general $l(D) \neq L_{R}(D)$ even if $R=\mathbf{Z}$. A simple example is $U_{3}(k)$ (see 6.1);

(i) $l\left(U_{3}(k)\right)=\mathbf{Z}^{2} \oplus \mathbf{Z} / k \oplus \mathbf{Z}$ generated as an abelian group by $u_{12}, u_{23}, u_{13}, z$ respectively, where $z$ is the class of $u_{13}^{k}$. The only nonzero bracket is $\left[u_{12}, u_{23}\right]=z$,

(ii) $L_{\mathbf{Z}}\left(U_{3}(k)\right)=\mathbf{Z}^{3}$ generated by $u_{12}, u_{23}, u_{13}$ with $\left[u_{12}, u_{23}\right]=k u_{13}$ the only nonzero bracket.

Another important difference is that in general $L_{R}(D)$ is not graded. Consider, for example, the 7 dimensional nilpotent Lie algebra $\mathcal{G}$ over $\mathbf{R}$ with multiplication table

G:

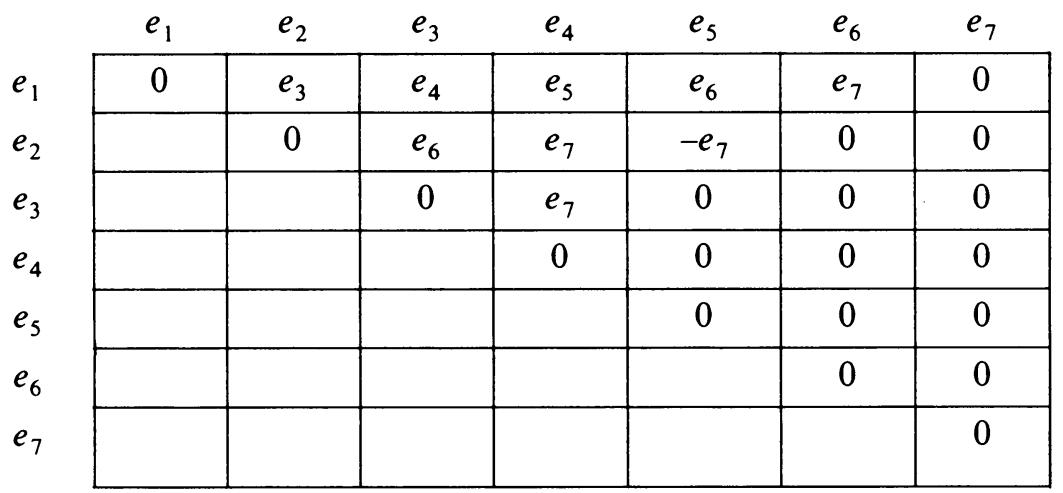

Let $G$ be the Baker-Campbell-Hausdorff group corresponding to $\mathcal{G}$. That is, $G=\mathcal{G}$ as manifolds and the product on $G$ is given by $x \cdot y=\rho(x, y)$ where $\rho(x, y)=x+$ $y+\frac{1}{2}[x, y]+\frac{1}{12}\{[x,[x, y]]-[y,[x, y]]\}+\cdots$ is the Baker-Campbell-Hausdorff formula [S]. Since the structure constants of $\mathcal{G}$ are rational, there is a discrete uniform subgroup $D \subset G$ by Malcev's results [M]. By the Lemma of $1.9, L_{R}(D) \otimes \mathbf{R}$ $\approx \mathcal{G}$; however it is easy to see that $\mathcal{G}$ cannot admit any nontrivial grading so neither can $L_{R}(D)$. 
In the case of the unipotent group $U_{n}(Z)$ we have

Proposition. $L_{\mathbf{Z}}\left(U_{n}(\mathbf{Z})\right)=l\left(U_{n}(\mathbf{Z})\right)$ as Lie algebras over $\mathbf{Z}$.

Proof. Using the canonical basis $\left\{u_{i j}: 1 \leqslant i<j \leqslant n\right\}$ of the example in 1.5 as a Z-basis for $L_{\mathbf{Z}}\left(U_{n}(\mathbf{Z})\right)$ we have

$$
\left[u_{i j}, u_{k l}\right]=b\left(u_{i j}, u_{k l}\right)-b\left(u_{k l}, u_{i j}\right)= \begin{cases}u_{i l} & \text { if } j=k, \\ -u_{k, j} & \text { if } i=l, \\ 0 & \text { otherwise. }\end{cases}
$$

On the other hand, the lower central series agrees with the upper central series in this case and we have the commutator relation

$$
\left(u_{i j}, u_{k l}\right)=u_{i j} u_{k l} u_{i j}^{-1} u_{k l}^{-1}= \begin{cases}u_{i l} & \text { if } j=k, \\ u_{k, j}^{-1} & \text { if } i=l, \\ 1 & \text { otherwise. }\end{cases}
$$

Hence $l\left(U_{n}(\mathbf{Z})\right)$ also has $\left\{u_{i j}: 1 \leqslant i<j \leqslant n\right\}$ as Z-basis and satisfies the same bracket relations.

\section{REFERENCES}

[C] H. Cartan, Théories cohomologiques, Invent. Math. 35 (1976), 261-271.

[CE] H. Cartan and S. Eilenberg, Homological algebra, Princeton Univ. Press, Princeton, N. J., 1956.

[CP] B. Cenkl and R. Porter, De Rham Theorem with cubical forms (preprint).

[Cv] C. Chevalley, Theory of Lie groups, Princeton Univ. Press, Princeton, N. J., 1946.

[G] W. Grueb, Multilinear algebra, Universitext, Springer-Verlag, New York, 1978.

[H] P. Hall, Nilpotent groups, Canad. Math. Congress, Edmonton, 1957 (reissued by Queens College, London).

[Hi] G. Hirsch, L'anneau de cohomologie d' un espace fibré en spheres, C. R. Acad. Sci. Paris Sér. A-B 241 (1955), 1021-1023.

[Hu] J. E. Humphreys, Linear algebraic groups, Springer-Verlag, New York, 1975.

[Lz] M. Lazard, Sur les groupes nilpotents et les anneaux de Lie, Ann. Ecole Norm. Sup. 71 (1954), $101-190$.

[Mc] S. Mac Lane, Homology, Die Grundlehren der math. Wissenschaften, Band 114, Springer-Verlag, New York, 1963.

[MB] S. Mac Lane and G. Birkhoff, Algebra, Macmillan, New York, 1971.

[M] A. Malcev, On a class of homogeneous spaces, Izv. Akad. Nauk SSSR Ser. Mat. 13 (1949), 9-32; English transl., Math. USSR-Izv. 39 (1949).

[Mi] E. Miller, DeRham cohomology with arbitrary coefficients, Topology 17 (1978), 193-203.

[N] K. Nomizu, On the cohomology of compact homogeneous space of nilpotent Lie groups, Ann. of Math. (2) 59 (1954), 531-538.

[Q] D. Quillen, On the associated graded ring of a group ring, J. Algebra 10 (1968).

[S] J. P. Serre, Lie algebras and Lie groups, Benjamin, New York, 1965.

[St] R. Steinberg, Lectures on Chevally groups, Yale University Lecture Notes, 1967.

[ST] H. Shulman and D. Tishler, Leaf invariants for foliations and the Van Est isomorphism, J. Differential Geom. 11 (1976), 539-546.

[Su] D. Sullivan, Infinitesimal computations in topology, IHES Publ. Math. 47 (1977), 269-331.

[V] V. Varadarajan, Lie groups, Lie algebras and their representations, Prentice-Hall, Englewood Cliffs, N. J., 1974.

Department of Mathematics, Michigan State University, East Lansing, Michigan 48824

Department of Mathematics, Northwestern University, Evanston, IllinOIS 60201 